\title{
A research based on testing method for the whole substation cascaded in digital substation
}

\author{
Yuekang $\mathrm{Wu}^{1}$, Shuheng Chen ${ }^{1}$, Shi Jing $^{1}$ and Xueting $\mathrm{Shi}^{2}$ \\ ${ }^{1}$ Sichuang Provincial Key Lab of Power System Wide-Area Measurement and Control, School of Mechanical and Electrical \\ Engineering, University of Electronic Science and Technology of China (UESTC), Chengdu 611713, China \\ ${ }^{2}$ Sichuang Provincial Key Lab of Power System Wide-Area Measurement and Control, School of Mechanical and Electrical \\ Engineering, University of Electronic Science and Technology of China (UESTC), Chengdu 611713, China
}

\begin{abstract}
Substation as an important component of the power grid, its safe and steady operation is crucial to the power grid. After building new substation or renovating the old ones, they needs to be tested to ensure its safe operation. The method, using relay protection tester to test the substation equipment nowadays, is not available to the whole cascade related connecting equipment, and the test work need a lot of manpower and material resources. To make up for all kinds of shortcomings when testing, this paper proposes a digital substation-oriented testing method for whole station concatenation, and established a testing software to test the whole cascaded substation, especially the method can realize closed-loop simulation test for whole station. In this method, the potential faults of digital substations can be predicted by using electromagnetic transient simulation to simulate various faults of substations, and the state of primary equipment can also be tested. The testing time can be shorten and the accuracy of testing can be improved, as well as, the safe and steady operation of substation equipment can be ensured by using the method proposed in this paper.
\end{abstract}

\section{Introduction}

With the development of economy, the power grid plays an important role in the whole society. In the six departments of power generation, transmission, transformation, distribution, power consumption and dispatching, transformation department occupies a very critical position when smart grid is becoming a trend of China's power grid. As the important part of the construction of strong smart grid, digital substation has been greatly developed in recent years [1]. According to the smart grid planning report of the State Grid Company: "equipment information, operation and maintaining strategy are fully interactive with power dispatching, realizing the state- based, comprehensive and optimized management of life cycle. The hub and central substations are built or transformed into digital, intelligent substations, which realize the unified data collection, real-time information sharing, real-time control and intelligent regulation of the whole network.

They also support the safe and stable operation of all levels of power grid and various advanced applications" [2]. So it is particularly important to carry out correct and complete testing of digital substations.

At present, few of the methods, used in conventional substation testing with relay protection device, have realized the complete test of the organization, distribution and transmission of the input signal of the protective device, as well as the output signal [3-4]. The testing method for relay protection device has some defects which needs to be improved, and compared with [5-6], almost none of method uses the closed-loop testing method to do the test for digital substation. The drawbacks of testing ways may lead to technical problems in the construction step of digital substations, which threaten the operation safety of digital substations, and lead to major equipment accidents in some serious cases. Therefore, simulating and predicting different variety of possible and potential faults can ensure the safety and steady operation of digital substation, at the same time the check of digital substation equipment is also the key to ensure the substation operate normally. This paper proposed a method and established a software platform which can be simulated and tested in steady and transient state focusing on whole station cascaded digital substation. The platform, acting as the main station of the simulation system, carries on the data communication with each wireless sub-station, simulates the data uploading of collector simulator of the primary system, and realizes support of the switch configuration and on-off state reading synchronously, so that it can realize the integrity of the digital substation testing include secondary system. 


\subsection{System construction}

The main idea of the testing method, proposed in this paper, is to treat the secondary system of the digital substation which is tested as a whole, and also to bring the merge unit (MU) and the network switch into the detection scope for maintain the integrity of the secondary system connection while testing. The testing method will utilize testing simulation platform which established by $\mathrm{C}++$ language and the algorithm is based on method of Electro-Magnetic Transient Program (EMTP) to simulate the running status of the connection line in digital substation and transmit the simulation result to the collector simulator through wireless transmission. Also, synchronous injection of experimental signal to the MU of tested substation by multiple collector simulators with distributed layout is adopted in this method. This method also will use the intelligent switch box to return the statues of breakers to collectors. Then the status will be backed to the platform in order to check on/off statues of relay protection device under fault condition. Eventually this method can check the correctness of organized information, the distributed function of MU and network switch by using the network analyser, fault oscillography and the monitoring system of substation secondary system.

The testing system shown in Fig. 1 can be used to test the whole performance of the secondary system of digital substation and test the behaviour of relay protection devices.

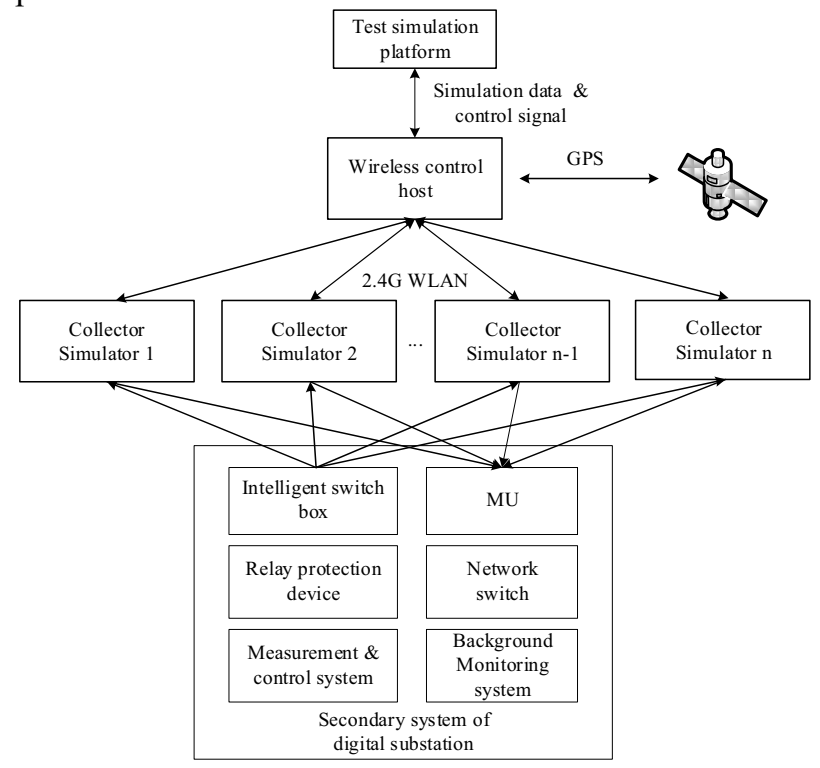

Fig. 1. System synchronization and overall test diagram.

The testing system of digital substation showed above can realize the accuracy of testing. The key factor of this process is to transmit the time domain simulation data to secondary system simultaneously. In order to realize the experimental scene close to the actual operation scene of digital substation, the data of any time period obtained by each MU must be synchronized, and the time interval between the two segments must also comply with the sampling rate requirement of the MU. At the same time, the accuracy of switching behaviour which intelligent switch boxes act depends on the synchronous clock. In this paper, the wireless control host provides synchronous clock to each collector simulator through Global Position System (GPS) timing [7-9]. This paper makes a long-distance experiment of the synchronous clock so that it can simulate the distance between the real substation monitoring room and the testing position. The test indicates that the time difference is within 10 minutes between the distance, which is wireless host to each sub-terminal, of 50-70 and 5-10 meters, while the speed of date uploading are the same.

\subsection{Introduction to testing method}

The whole station cascaded testing method of digital substation is a digital substation integrated performance testing technology, based on power network simulation, wireless synchronization, transmission and decentralized injection. The main function of the method is to treat the secondary system of digital substation as a whole. The method not only test the function of digital substation equipment, but also make the MU and network switch whose function can take the organization and distribution of information in digital substation into the testing scope completely. In order to test the overall performance of the digital substation, it's necessary to maintain the integrity of the connection and the input information while testing the secondary system of digital substation. This test can realize the electromagnetic transient simulation of digital substation and the result can be sent to each sub-terminal in wireless form. Then the terminal transmits the information to the MU through optical fibre, thus completing the integrity performance test of the secondary system of digital substation. The test system includes the notebook computer, the substation simulation platform, the wireless control host and the collector simulators.

The flow chart of the test method is shown in Fig. 2.

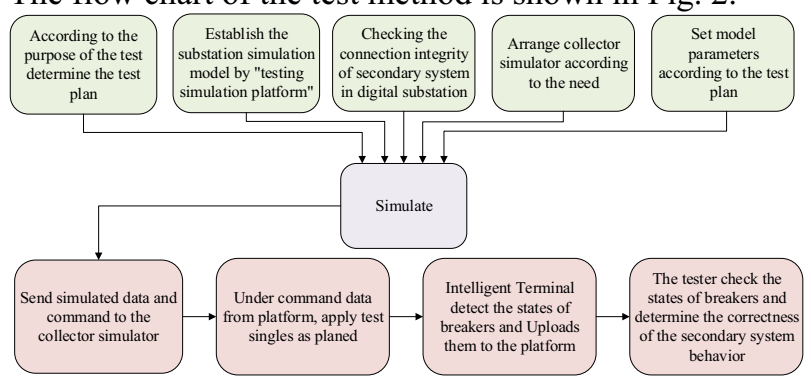

Fig. 2. Flow chart of the test method.

The method can improve the efficiency of the system testing as well as ensure the integrity of the system testing. The purpose of the test is designed and the method is determined in advance, according to the scheme, the corresponding model and parameters are set up on the testing simulation platform. The collector simulators will be reasonably arranged depending on the actual needs of the scheme, and then the simulation will be initiated. The data will be injected into each simulators synchronously after simulation. When the operations mentioned above have been finished, the test will start, the operation will be carried out rely on the presetting control instruction, and then the parameters 
and states will be collected and transmitted back to the testing simulation platform. The tester will determine the validity of the secondary system behaviour according to the action behaviour of the intelligent terminal.

\subsection{Closed-loop simulation}

At present, most of the relay protection testing devices used in China are protective tester [10]. It still stays in the test stage of open loop on single device, and can only complete the basic function test of protection device. However, it is hard to test the whole performance of power system equipment, to carry out the closed-loop test, as well as the system-level test of relay protection device. Hence, in order to improve the stability and reliability of secondary equipment in substation, it is necessary to study the closed-loop system test for secondary equipment in substation. Especially for the digital substation based on IEC 61850 [11], It's vital that the test for the closed-loop system of relay protection device must be done. The method proposed in this paper are fit for system-level, which can do the basic function test, on single unit, and systematic test simultaneously. It can also ensure that the software and the hardware of whole substation work well. The closed-loop for simulation is shown in Figure. 3:

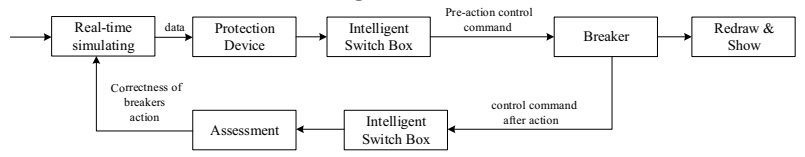

Fig. 3. Closed-loop simulation.

The simulation platform carry out simulation by the given parameters and setting, after that it will upload the result to protection device, then the protection device then transfers the switch action instructions to the intelligent switch box, the breaker acts according to the pre-action control command which is uploaded by the intelligent switch box. When the action completes, the platform will redraw the breaker state, meanwhile, the action statues will be returned back to the intelligent switch box, box will continually send the data back to assess. In assessment part, people will judge whether the acting of breakers is right. If the breakers have done incorrectly, the assessment segment will give the alarm to tell people which breaker is wrong and find out the causes. Then it will send the right statues of breakers to the platform for simulation again in order to do the correct experiment. Hence the closed loop have been achieved.

Through closed-loop simulation, it is possible to test the whole system and avoid under the condition of only testing the performance on a single device. At the same time, the whole information transmission loop should also be involved in the test scope, which can quickly find out the secondary equipment fault and feedback to the testers. For example, data transmission problems like damaging optical fibre which cause faults like device protection locking and unusual action and so on, it will be quickly found and warning message will be informed instantly. Optical cables have been used for signals transmitting in digital substation instead of the power cables, hence it's crucial to test the secondary equipment and secondary circuits, including optical fibre links. By adopting the testing method provided in this paper, the electric devices can be quickly tested according to the feedback result as well as information transmission loop. Meanwhile, the efficiency can be improved, the system testing becomes more comprehensive.

\section{Transient model establishment}

With the gradually increase of power grid voltage level and the capacity, the transient process decay time increases, and the influence of transient components on the protection operation becomes more and more serious. Because the conventional protection inspection instrument can't simulate the transient components, it can only be limited to the conventional test of the protection device, and can't analyse the results of the protection operation well. In the past, the test of the action behaviour of the protective device is also done by using the dynamic simulation, which can only be done indoors and can't be applied to the real substation, so the expansibility and flexibility are relatively low. But the computer technology develops quickly, it is possible to get transient simulation of power system by computer, and the transient simulation results can be output smoothly, therefore, in this paper it will use numerical method to calculate the electromagnetic transient.

In view of the fast changing speed of electromagnetic transient process, it is generally necessary to calculate and analyse the transient values of voltage and current in milliseconds. Numerical integration algorithm is the basic method of power system transient stability analysis. In this paper, the element model is analysed and the element transient equivalent model is established by numerical integration method.

The main numerical methods for power system stability calculation are Euler method, Runge-Kutta method, predictive correction and trapezoid method for solving differential equations, Newton method for solving algebraic equations and iterative solution for triangular decomposition, etc. The numerical integration method of differential equation can be divided into two kinds: explicit integration algorithm and implicit integration algorithm.

For a set of equations

$$
\left\{\begin{array}{c}
\frac{d Y}{d t}=F(Y, t) \\
Y\left(t_{0}\right)=Y_{0}
\end{array}\right.
$$

When we use explicit integration algorithm to calculate, we can calculate the algebraic equations and differential equations separately, only use the value at $t-\Delta t$ when we calculate the value at $t$, this method can easy programming and convenient expanding, but because of the existence of interfacing error between the differential equation and algebraic equation, the stability of integral calculation is not high. Implicit integration method can maintain the stability of integral calculation very well. Using ordinary differential equation 
$y^{\prime}=-y+x+1$ to show the difference of calculation stability, this method can also be improved in nonliner ordinary differential equation, but in this paper will not describe in details.

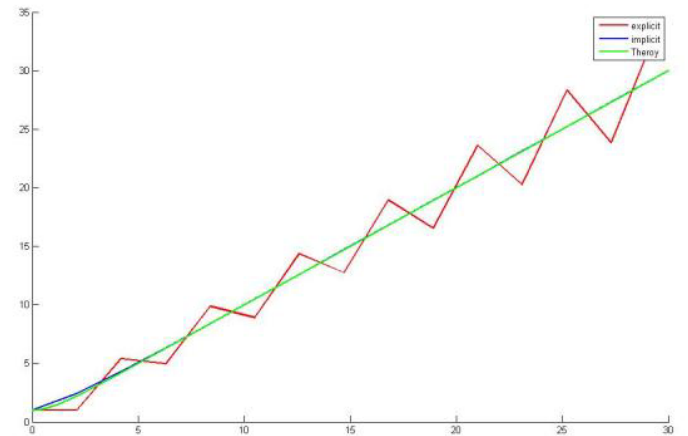

Fig. 4. Calculation stability of explicit and implicit integration algorithm.

In Figure. 4 we can see that explicit integration become deviate the theoretical value with increase, and on the contrary implicit integration algorithm become more and more close to the theoretical value. Figure. 4 can verify the result that using implicit integration algorithm can get well calculation stability and get good performance.

At the same time, the truncation error caused by Euler method and trapezoidal method is different, Euler method has the first order precision and the trapezoidal method has the second order precision. Therefore, the trapezoidal formula of implicit integral is used to differentiate the differential equation, and the differential equation and network equation are used to get the calculation result.

For example, the open loop transfer function like:

$$
\frac{7 s+100}{s^{4}+7 s^{3}+28.44 s^{2}+65.11 s}
$$

Given unit step signal to this system, getting the output curve of closed-loop transfer function.

Figure. 5 is the precision difference of Euler and trapezoid method:

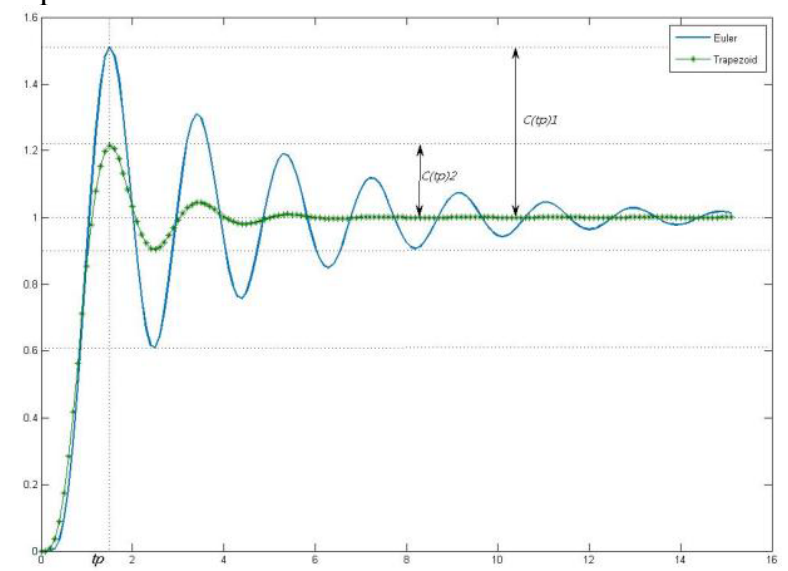

Fig. 5. Precision difference of Euler and trapezoid.

From Figure.5 we can see that the maximum overshoot value of Euler method is higher than Trapezoid method, and the response speed of trapezoidal method to steady state is much more faster than that of Euler method. The oscillation time and amplitude of
Euler method are larger than that of trapezoidal method. In the numerical calculation process, there are other algorithms with higher accuracy like RK algorithm and Simpson algorithm, but compared with implicit trapezoidal integration method, there are not easy to program and the implicit trapezoidal integration method can meet the accuracy in real situation. Therefore, the implicit trapezoidal integration method is used to calculate the electromagnetic transient process, which can meet the speed of computation and meet higher accuracy.

Based on the core idea of implicit trapezoidal integral method, the algebraic difference equations of the common components of each circuit are derived one by one, and the corresponding transient equivalent model is obtained, which provides the model basis for subsequent calculation. In this paper, implicit trapezoidal integral method had been used to get the three-phase resistance, inductor series coupling circuit as an example:

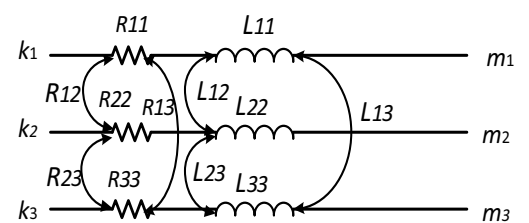

(a)

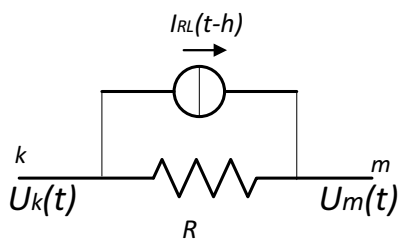

(b)

Fig.6. (a) is the three-phase resistance, inductor series coupling circuit.(b) is the transient equivalent model of (a).

If the three-phase circuit is balanced, the differential equations are listed according to the law of electromagnetic induction.

$$
R i_{k m}(t)+L \frac{d i_{k m}(t)}{d t}=u_{k}(t)-u_{m}(t)
$$

In this equations $k$ is represented $k_{1}, k_{2}, k_{3}$ and $m$ is represented $m_{1}, m_{2}, m_{3}$.

Using the trapezoidal integral formula to differencing the differential equation can get:

$$
i_{k m}(t+h)=S^{-1}\left[u_{k}(t+h)-u_{m}(t+h)\right]+I_{k m}(t)
$$

In equation (4)

$$
\begin{gathered}
S=R+\frac{2 L}{h} \\
I_{k m}(t)=S^{-1}[S-2 R] i_{k m}(t)+S^{-1}\left[u_{k}(t)-u_{m}(t)\right]
\end{gathered}
$$

$$
\begin{aligned}
& \text { Also according to formula (6) can get } \\
& \qquad \begin{aligned}
I_{k m}(t)= & H\left[u_{k}(t)-u_{m}(t)+S I_{k m}(t-h)\right] \\
& -I_{k m}(t-h)
\end{aligned}
\end{aligned}
$$

In equation (7)

$$
H=2\left[S^{-1}-S^{-1} R S^{-1}\right]
$$


In the formula (8) showed above, $S$ is a resistance matrix

$$
S=\left[\begin{array}{ccc}
R_{s} & R_{m} & R_{m} \\
R_{m} & R_{s} & R_{m} \\
R_{m} & R_{m} & R_{s}
\end{array}\right]+\frac{2}{h}\left[\begin{array}{ccc}
L_{s} & L_{m} & L_{m} \\
L_{m} & L_{s} & L_{m} \\
L_{m} & L_{m} & L_{s}
\end{array}\right]
$$

In the above

$$
\begin{cases}R_{s}=\frac{1}{3}\left(R_{0}+2 R_{1}\right) & R_{m}=\frac{1}{3}\left(R_{0}-R_{1}\right) \\ L_{s}=\frac{1}{3}\left(L_{0}+2 L_{1}\right) & L_{m}=\frac{1}{3}\left(L_{0}-L_{1}\right)\end{cases}
$$

$L_{s}$ is the self-inductance and $L_{m}$ is the mutualinductance, $R_{s}$ is the self-resistance and $R_{m}$ is the mutual-resistance, $L_{1}$ and $R_{1}$ are the positive sequence inductor and resistance, $L_{0}$ and $R_{0}$ are the zero sequence inductor and resistance, and $H$ is admittance matrix, due to the existence of coupling, they are all full array. If there is no resistance in the coupling branch, that is, $R$ is a zero matrix, in this moment
$S=\frac{2 L}{h}, H=\frac{h}{2 L} \quad$, it's a three-phase balanced coupling inductor circuit.

According the implicit trapezoidal integral method can get all transient equivalent model of common electrical components, and then get the admittance matrix , according the admittance matrix iteration can do the transient calculation and get the calculate result.

\section{Result}

The digital substation-oriented testing method, mentioned in the thesis, and the established system can obtain an electrical logic model. This model is able to do the numerical simulation and obtain the data which come from the steady-state operation and fault transient system. Moreover, it can offer the instant feedback to the platform operators as well while simulates operatingstate of digital substation. It's obvious that the model could lay the foundation for providing more humanized man-machine interface.

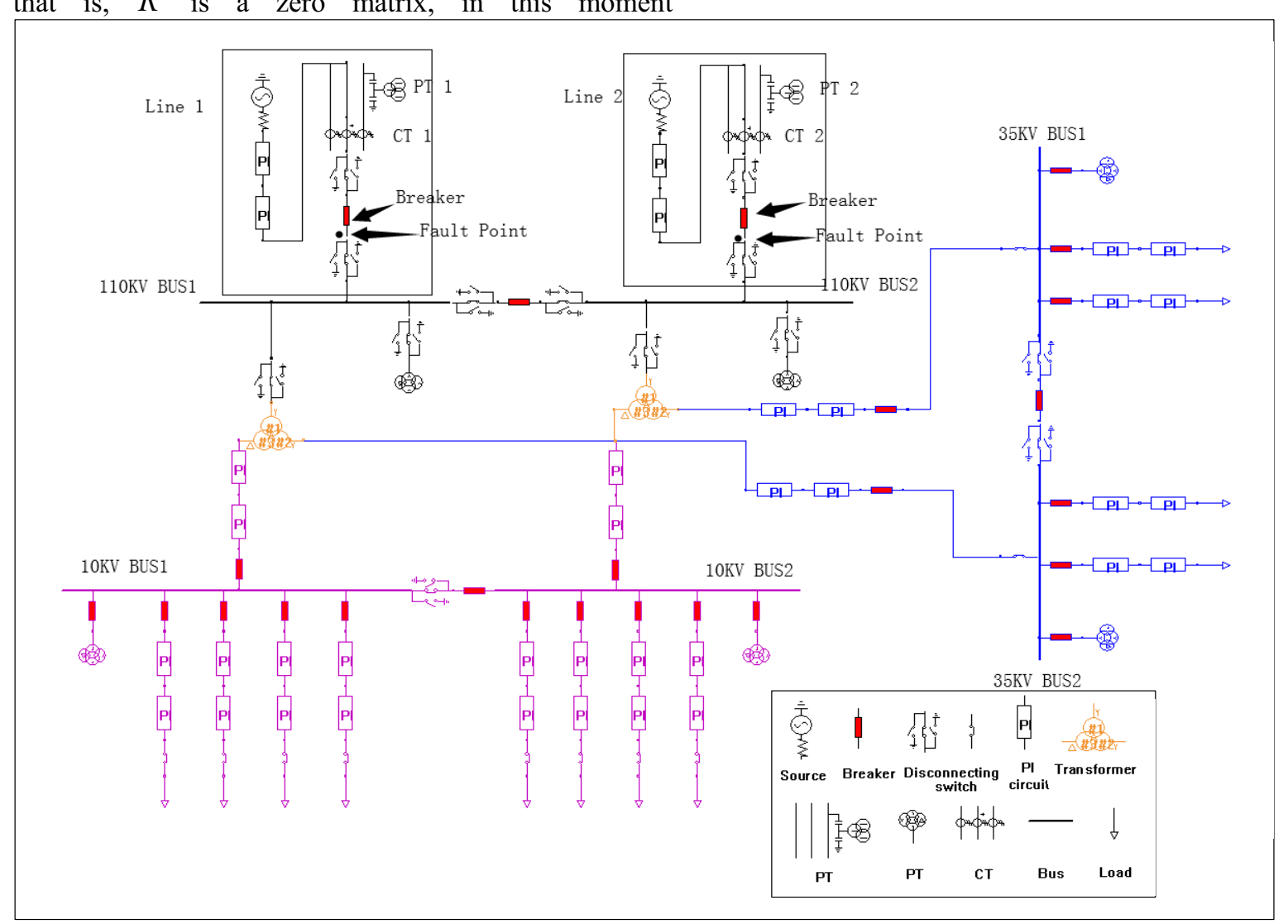

Fig. 7. A digital substation under test.

The system based on the method which is proposed in this paper, have been used in a substation of China Southern Power Grid. For instance, the traditional fault of substation such as single-phase transient grounding fault and three-phase transient grounding fault can be simulated. The single-phase transient fault and the threephase transient grounding fault are set to a $110 \mathrm{KV}$ substation line 1 and line 2 respectively in Figure. 7. In Figure. 7, the Fault Point is the point where set fault and $\mathrm{t} 1$ is the time when fault initiated, and $\mathrm{t} 3$ was the ending time, the simulating result can be seen in Figure. 8 and Figure. 9 respectively.

\subsection{Single-phase transient grounding fault}


At time t1, a fault is applied to the line 1 of phase A and ends in time $\mathrm{t} 3$. Owing to the delay of control command, the breaker will work till t2, and end in t4. During the time of fault, Phase A is grounded, which causes the voltage becoming zero. The current amplitude of phase A increase slightly as well as the other two phase voltages. At the same time, the statue of breakers also show in the Figure. 8.

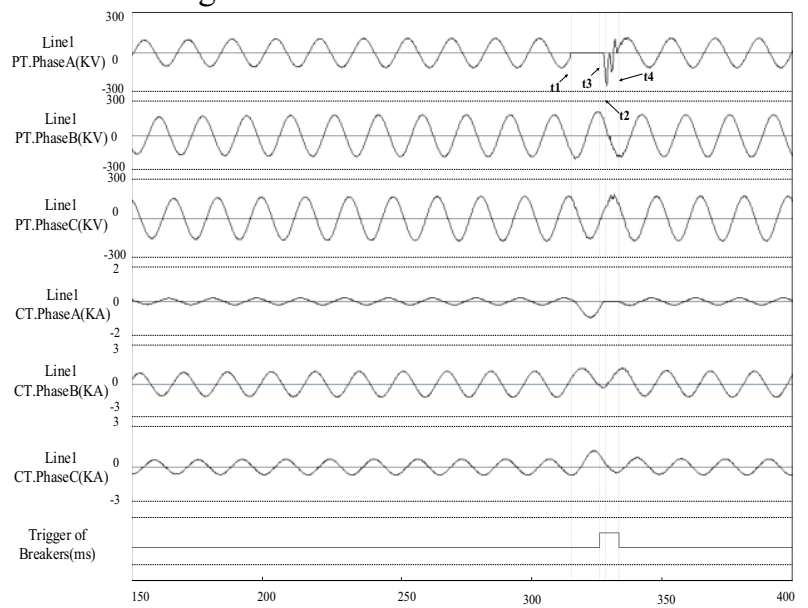

Fig. 8. Single-phase Transient Fault of Line 1 Phase A.

\subsection{Three-phase transient grounding fault}

At time $\mathrm{t}$, a three-phase transient grounding fault is applied to the three phase of line 1 and fault ends in time t3 . Owing to the delay of control command, the breaker will act till $\mathrm{t} 2$. During the time of fault, the voltages of phase A, B, C all become zero because of grounding. The statue of breakers is shown in Figure. 9.

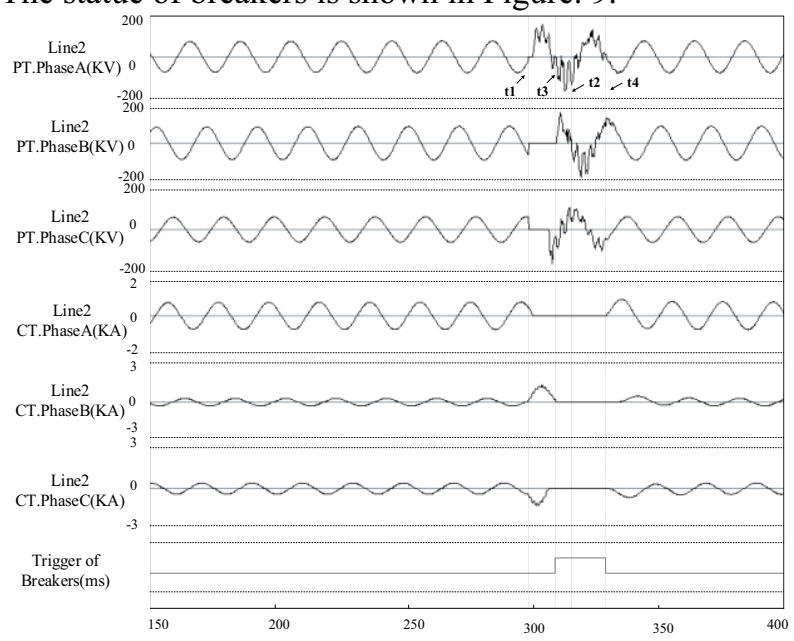

Fig. 9. Single-phase Transient Fault of Line 1 Phase A, B, C.

Through the two common faults mentioned above, it is obvious that the system established, using this method, can truly simulate the real substation faults and fulfil the requirements of the real substation transient simulation.

\subsection{Field application}

The method proposed in this paper and the established software has applied in a digital substation in China Southern Power Grid, and has verified the correctness of function. Figure.10 and Table. 1 are the result when the simulate platform give the simulate result to a line of $10 \mathrm{KV}$ capacitor which its current is $35.0 \mathrm{~A}$ and the time of duration is $650 \mathrm{~ms}$, the protection measurement and control device displays the over current I and II segment action, and showing the information of breakers (the I, II and III segment protective setting is $33.3 \mathrm{~A} 0 \mathrm{~ms}, 11.5 \mathrm{~A}$ $200 \mathrm{~ms}$ and $3.47 \mathrm{~A} 500 \mathrm{~ms}$ ) trip operation.

Table. 1 Results of protection test in field application

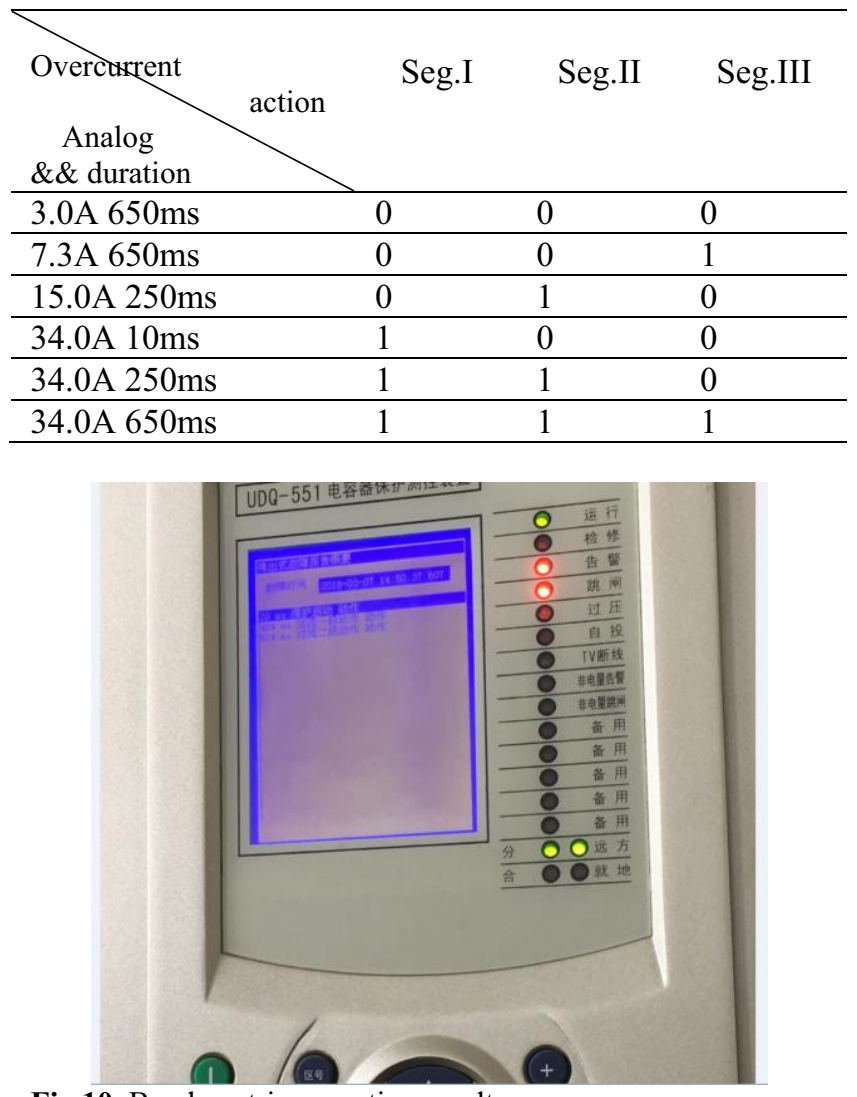

Fig.10. Breakers trip operation result.

\subsection{Time of synchronization}

Through the wireless synchronization function, the substation injecting data synchronously can be realized, the simulation system can send the same sets of data to the collector simulators.

By sending the same sets of data to the collectors and then gather the data to $\mathrm{MU}$, the phase difference of data collected by different collector terminals is about $0.08^{\circ}$, while the time is about 4us. That is to say, the time difference between wireless host and each terminal is 4us, within a reasonable range and can be termed as injected synchronously. The whole station cascaded testing method proposed in this paper and the system established by using the unified clock and data synchronous upload in each department can perform well. It represents the real situation for simulating substation.

\section{Conclusion}

The traditional substations do a function test on the primary electrical equipment only though the giving 
analog value [12] which is unavailable to the substation equipment connected wholly. This method ignores the correctness of interconnection between devices and communication equipment such as optical fibre and network switch in substation. All devices only do conformance testing, in other words, this method only do the test for the inputting and outputting data and specification aspect testing, seldom do the functional tests under the whole station cascaded statues.

This paper proposes a cascade station method in digital substation test. Problems, like incomplete function test, potential fault detection and so on, can be avoided by this method. It can find out the potential faults of digital substation effectively, not only limited to single device performance test, but also to the whole digital substation for system-level testing. At the same time, the system-level test results can also ensure that the functions of the secondary equipment like the relay protection devices, the measurement and control device are normal. The problems of traditional substation test have been solved. The method mentioned in this thesis avoids the separation of communication and devices and improves the test efficiency dramatically.

\section{Announcement}

Supported by "The Fundamental Research Funds for the Central Universities" (ZYGX2015J105) for sponsoring this research.

\section{References}

1. Dechang Yang, Li Yong, C.Rehtanz, et al. Research on the composition and development planning of Chinese smart grid. J. power grid technology,(2009), 33 (20): 13-20.

2. Jun Feng. Principle and Test Technology of Digital Substation. M. China Electric Power Press (2011).
3. Gao Xiang, Zhang Peichao. Main features and key technologies of digital substation. J. power grid technology (2006) 30 (23): 67-71.

4. Li Xianmei, Huang Jiadong, Tang Baofeng. Analysis and research of relay protection testing technology in digital substation. J. power system protection and control (2012) 40 (3): 105-108.

5. J. Bowen, "Substation commissioning and turnover planning," IEEE Ind. Appl. Mag., vol. 6, no. 2, pp. 8-22, Mar./Apr(2000).

6. Wu Zai-jun, Hu Min-qiang. Analysis of IEC61850communication networks and system in substations. J. Electric Power Automation Equipment (2002).

7. Yu Pengfei, Yu Qiang, Deng Hui, et al. Application scheme of IEEE 1588 precise time synchronization protocol. J. automation of electric power system (2009), 33 (13): 99-103.

8. Zhao Shanglin, Hu Minqiang, Dou Xiaobo, et al. Research on clock synchronization technology in digital substation based on IEEE1588. J. power grid technology, (2008) 32 (21): 97-102.

9. Jing $\mathrm{S}$, Huang $\mathrm{Q}, \mathrm{Wu} \mathrm{J}$, et al. Wireless clock synchronization method for whole-view test of Intelligent Substation. J. Automation of Electric Power System, (2013) 37(9):103-109.

10. Daboul M, Orsagova J, Bajanek T, et al. Testing protection relays based on IEC 61850 in Substation Automation Systems. C. International Scientific Conference on Electric Power Engineering. IEEE, (2015):335-340.

11. Kaneda K, Tamura S, Fujiyama N, et al. IEC61850 based Substation Automation System. C. Joint International Conference on Power System Technology and IEEE Power India Conference, 2008. Powercon. IEEE, (2008):1-8.

12. Zhou Chunxia, Zhan Rongrong, Jiang Jianning, et al. $500 \mathrm{kV}$ digital substation dynamic model test research. J. Power grid technology, (2010) 34 (6): 193-197. 